\title{
Experimental Investigation of a Four-Dimensional 256-ary Lattice-based Modulation Format
}

\author{
Tobias A. Eriksson ${ }^{1}$, Saleem Alreesh ${ }^{2,3}$, Carsten Schmidt-Langhorst $^{3}$, Felix Frey ${ }^{3}$, \\ Pablo Wilke Berenguer ${ }^{3}$, Colja Schubert ${ }^{3}$, Johannes K. Fischer ${ }^{3}$, \\ Peter A. Andrekson ${ }^{1}$, Magnus Karlsson ${ }^{1}$, and Erik Agrell ${ }^{4}$ \\ ${ }^{1}$ Dept. of Microtechnology and Nanoscience. ${ }^{4}$ Dept. of Signals and Systems. Chalmers University of Technology, \\ SE-412 96, Gothenburg, Sweden. \\ ${ }^{2}$ Technische Universität Berlin, Fachgebiet Nachrichtentechnik, Einsteinufer 25, 10587 Berlin, Germany. \\ ${ }^{3}$ Fraunhofer Institute for Telecommunications Heinrich Hertz Institute, Einsteinufer 37, 10587 Berlin, Germany. \\ tobias.eriksson@chalmers.se
}

\begin{abstract}
A four-dimensional modulation format with 256 point from the $\mathrm{D}_{4}$-lattice $\left(256-\mathrm{D}_{4}\right)$ is compared to PM-16QAM in experiments. $256-\mathrm{D}_{4}$ is more sensitive at high OSNR but with a $21.3 \%$ overhead turbo product code, PM-16QAM outperforms $256-\mathrm{D}_{4}$.

OCIS codes: (060.4080) Modulation, (060.2330) Fiber optics communications
\end{abstract}

\section{Introduction}

Polarization-multiplexed 16-ary quadrature amplitude modulation (PM-16QAM) is a well-studied modulation format that is often considered for systems where a higher spectral efficiency (SE) than PM quadrature phase shift keying (PMQPSK) is required. PM-16QAM doubles the SE compared to PM-QPSK and can be generated with low complexity. However, the sensitivity of PM-16QAM is reduced compared to PM-QPSK which lowers the achievable transmission distances. Investigation of modulation formats that span over the four dimensional (4D) field has revealed formats with increased sensitivity and with SEs close to that of PM-16QAM [1,2]. These formats are often characterized using the power efficiency (PE), which gives the sensitivity gain over QPSK at asymptotically high optical signal to noise ratio (OSNR) [1]. One such 4D format is 128-ary set-partitioning QAM (128-SP-QAM) which has $2.43 \mathrm{~dB}$ increased PE over PM-16QAM at the cost of $0.5 \mathrm{bit} / \mathrm{symbol} /$ polarization lower SE and has in experiments been shown to achieve more than $50 \%$ increased transmission distance compared to PM-16QAM [3]. 512-ary set-partitioning QAM (512SP-QAM) has $0.3 \mathrm{~dB}$ better PE than PM-16QAM and 0.5 bit/symbol/polarization increased SE [4]. In experiments this format has been shown to have $45 \%$ reduced transmission distance compared to PM-16QAM and $50 \%$ increased distance over PM 32-ary QAM [5].

In this paper we study a modulation format that uses 256 points from the $\mathrm{D}_{4}$-lattice $\left(256-\mathrm{D}_{4}\right)$ for coherent optical communication systems. This modulation format has the same SE of 4 bits/symbol/polarization as PM-16QAM and a PE of $-2.27 \mathrm{~dB}$ which is an increase of $1.71 \mathrm{~dB}$ compared to PM-16QAM. This is, to our knowledge, the most power efficient known 4D format with 256 points. We compare 256- $\mathrm{D}_{4}$ with PM-16QAM in 28 GBd WDM experiments showing that $256-\mathrm{D}_{4}$ is a promising format for uncoded systems or systems employing low overhead, low complexity forward-error correction (FEC) operating at BER $\leq 10^{-3}$. However, at soft-decision FEC-targets of around BER $=10^{-2}$, PM-16QAM shows a superior performance compared to $256-\mathrm{D}_{4}$ in both back-to-back and long-haul WDM transmission experiments.

\section{The 256- $\mathrm{D}_{4}$ format}

The 256- $\mathrm{D}_{4}$ takes points from the $\mathrm{D}_{4}$ lattice, which is the same lattice as other $4 \mathrm{D}$ modulation formats such as 128 SP-QAM and 512-SP-QAM are based on [1,3-5]. This format has previously been studied theoretically [6,7] and

\begin{tabular}{|c|c|}
\hline $\begin{array}{c}7 \text { levels driving } \\
\text { signal expression }\end{array}$ & $\begin{array}{c}6 \text { levels driving } \\
\text { signal expression }\end{array}$ \\
$( \pm 1,0,0,0)$ & $( \pm 1, \pm 1, \pm 1, \pm 1)$ \\
$( \pm 1, \pm 1, \pm 1,0)$ & $( \pm 3, \pm 1, \pm 1, \pm 1)$ \\
$( \pm 1, \pm 2,0,0)$ & $( \pm 3, \pm 3, \pm 1, \pm 1)$ \\
$( \pm 1, \pm 1, \pm 1, \pm 2)$ & $( \pm 5, \pm 1, \pm 1, \pm 1)$ \\
$( \pm 1, \pm 2, \pm 2,0)$ & $( \pm 3, \pm 3, \pm 3, \pm 1)$ \\
$( \pm 3,0,0,0)$ & $( \pm 3, \pm 5, \pm 1, \pm 1)$ \\
& $( \pm 3, \pm 3, \pm 3, \pm 3)$ \\
\hline
\end{tabular}

Table 1. Two representations of $256-\mathrm{D}_{4}$ Left: Use all sign combinations and permutations. Right: Use all sign combinations and permutations whose coordinate sum is a muliple of 4 .

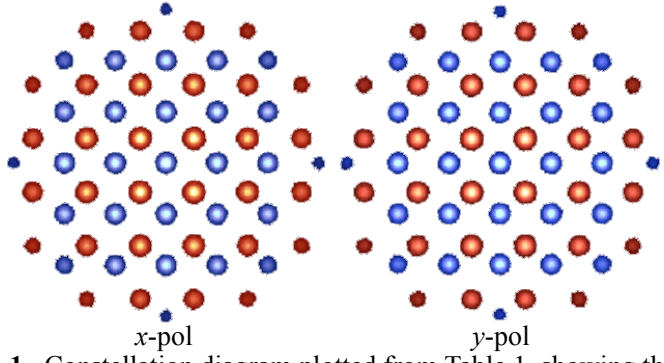

Fig. 1. Constellation diagram plotted from Table 1, showing the 7-level expression in blue and the 6-level driving signals expression in red. Note that the constellations have been normalized. Color grading indicates probability of occurance.

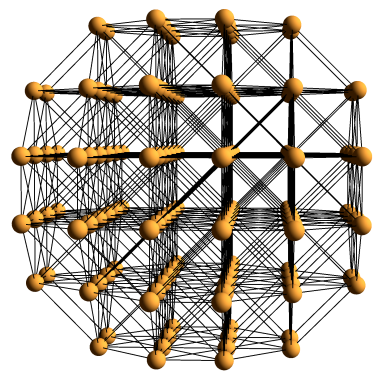

Fig. 2. 3D projection of the $256-\mathrm{D}_{4}$ format. 

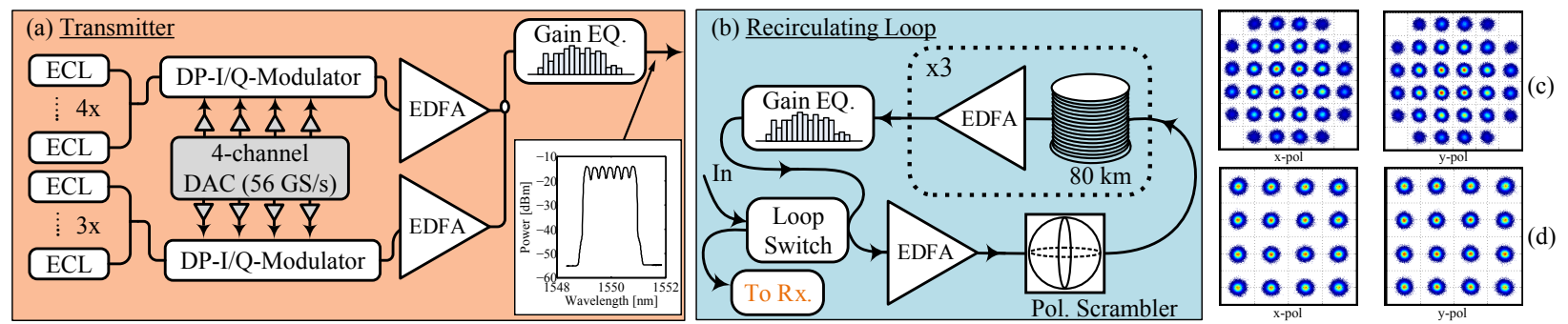

Fig. 3. (a) Experimental setup of the transmitter for both 256- $\mathrm{D}_{4}$ and PM-16QAM. (b) The recirculating loop consists of 3 spans of $80 \mathrm{~km}$ SSMF. (c) Measured back-to-back constellation for 256- $\mathrm{D}_{4}$ at $37.3 \mathrm{~dB}$ OSNR. (d) Measured back-to-back constellation for PM-16QAM at $37.4 \mathrm{~dB}$ OSNR.

is to our knowledge the most power efficient known modulation format with 256 points in four dimensions. In the context of bit-interleaved coded modulation, this format was studied in [8], and a similar format was experimentally investigated in [9]. The symbols of $256-\mathrm{D}_{4}$ are given in Table 1 for two different $4 \mathrm{D}$ rotations. Note that for the right column, only permutations and sign selections for which the sum of the coordinates is a multiple of 4 is allowed. This description is interesting from an implementation point of view since it requires one less level in the driving signals, when implemented using one I/Q-modulator per polarization. This is illustrated in Fig. 1 where the projection to the $x$ and $y$-polarization are shown for the two descriptions (color-coded red and blue). As seen, the first description requires 7 levels in the driving signal and the second version 6 levels. A 3D-projection of the format is shown in Fig. 2, note that some points are overlapping in this projection.

\section{Experimental Setup}

The transmitter is shown in Fig. 3(a). 7 external cavity lasers (ECLs) with $\leq 100 \mathrm{kHz}$ linewidth and with a channel spacing of $35 \mathrm{GHz}$ are used. The even and odd channels are modulated separately using two dual-polarization I/Qmodulators, driven by $28 \mathrm{GBd}$ signals from a four-channel digital-to-analog converter (DAC) with $56 \mathrm{GS} / \mathrm{s}$ sample rate and 8 bit nominal resolution. We use root-raised cosine pulses with a roll-off factor of 0.1 and apply digital signal pre-distortion to compensate for the frequency response and the nonlinear transfer characteristics of the transmitter components. Pilot-symbol sequences are periodically inserted for data-aided channel estimation and carrier frequency recovery, resulting in an overhead of $2.7 \%$ [10].

As FEC-coding we apply a turbo product code (TPC) with BCH codes as component codes with the properties of $\mathrm{BCH}(256,239) \times \mathrm{BCH}(128,113)$ to both formats [11] which gives an overhead of $21.3 \%$. The PM-16QAM constellation was Gray-mapped in each polarization, and for $256-\mathrm{D}_{4}$ we use a numerically optimized bit mapping. The individual bit streams are encoded and 4 frames are interleaved in each polarization for PM-16QAM and 8 frames in $4 \mathrm{D}$ for $256-\mathrm{D}_{4}$, before bit-to-symbol mapping.

The signals were propagated over a recirculating loop, Fig. 3(b), consisting of 3 spans of $80 \mathrm{~km}$ standard singlemode fiber (SSMF), Erbium-doped fiber amplifiers (EDFAs), a loop-synchronized polarization scrambler and a programmable gain-equalization filter to compensate for the gain-tilt of the EDFAs. The receiver consisted of an EDFA followed by an optical-bandpass filter with $0.26 \mathrm{~nm} 3 \mathrm{~dB}$-bandwidth to select the channel under test which is detected using a polarization-diverse optical hybrid with balanced photo-detectors. The signals are sampled using analog-todigital converters with sample-rates of $50 \mathrm{GS} / \mathrm{s}$ and $20 \mathrm{GHz}$ bandwidth.

The digital signal processing (DSP) is performed off-line starting with optical-frontend correction and chromaticdispersion compensation followed by data-aided carrier frequency recovery, channel estimation and frequency-domain equalization [10]. Carrier phase recovery is performed by a blind phase search algorithm, where pilot-assisted phase unwrapping is applied for cycle slip mitigation. An additional T-spaced $4 \times 4$ adaptive equalizer based on a decision directed least-mean square criterion compensates for residual timing skew. For uncoded transmission, minimum Euclidean distance demapping is performed and for the TPC, iterative soft-input/soft-output decoding is used followed by hard-decision [11].

\section{Experimental Results}

The back-to-back results are shown in Fig. 4(a) together with simulation results for the additive white Gaussian noise (AWGN) channel. The measured implementation penalty at BER $=10^{-3}$ was $1.6 \mathrm{~dB}$ for uncoded $256-\mathrm{D}_{4}$ and $1.3 \mathrm{~dB}$ for uncoded PM-16QAM. At a pre-FEC target of $\mathrm{BER}=2 \times 10^{-2}$ we measure a $1.0 \mathrm{~dB}$ sensitivity gain for PM-16QAM over 256- $\mathrm{D}_{4}$. After decoding of the TPC with 5 iterations we see that PM-16QAM with TPC has a $1.4 \mathrm{~dB}$ sensitivity 

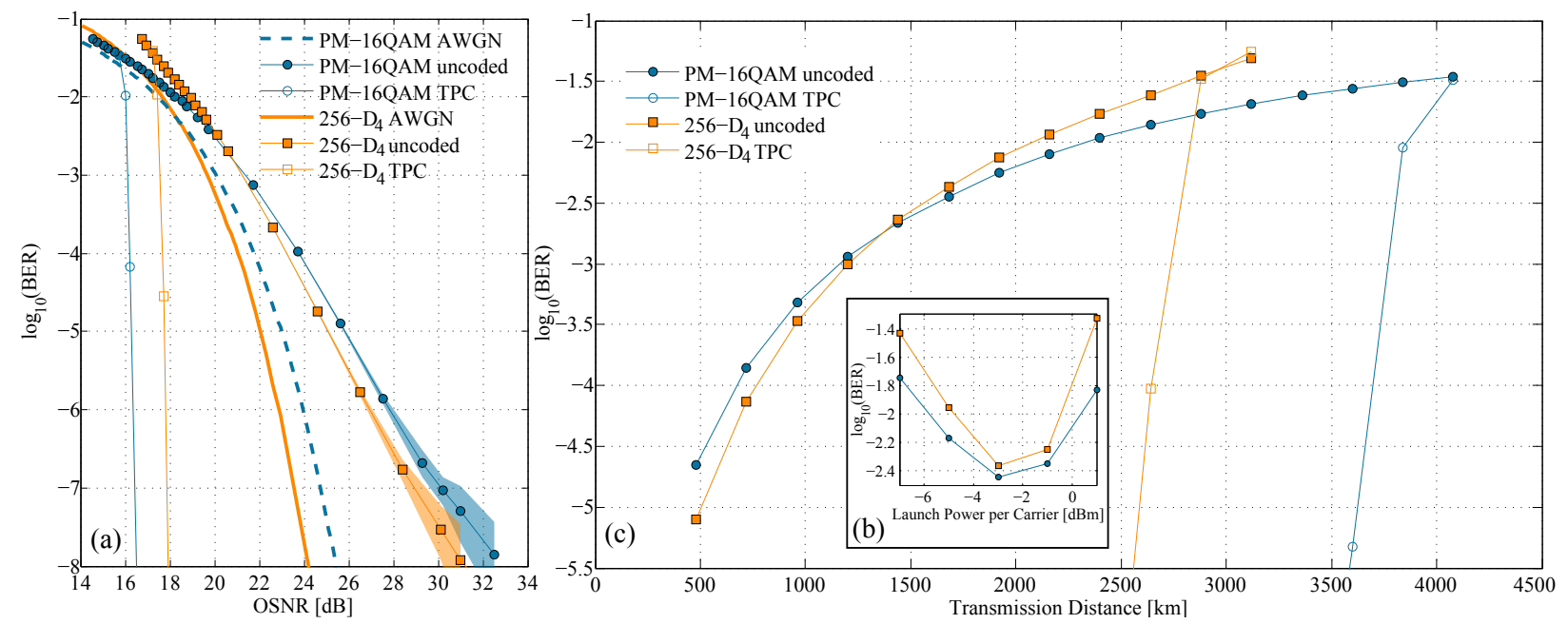

Fig. 4. (a) Back-to-back measurements of the center WDM-channel for uncoded (blue circles) and TPC (blue open circles) PM16QAM as well as uncoded (orange squares) and TPC (orange open squares) 256- $\mathrm{D}_{4}$. Shaded area shows the $99 \%$ confidence interval. Simulations with AWGN are also plotted for uncoded PM-16QAM (dashed blue line) and 256- D $_{4}$ (orange solid line). (b) BER as a function of launch power at $1680 \mathrm{~km}$ for uncoded 256- $\mathrm{D}_{4}$ and PM-16QAM measured for the center WDM-channel. (c) BER as a function of transmission distance for the center channel for all cases.

gain over 256- $\mathrm{D}_{4}$ with TPC. At lower BERs, 256- $\mathrm{D}_{4}$ is more sensitive than PM-16QAM and at BER $=10^{-6}, 256-\mathrm{D}_{4}$ has a $0.9 \mathrm{~dB}$ sensitivity gain over PM-16QAM. We process at least $2^{28}$ information bits per measurement point.

The BER as a function of optical launch power per carrier at a transmission distance of $1680 \mathrm{~km}$ for $256-\mathrm{D}_{4}$ and PM16QAM is shown in Fig. 4(b). The optimal launch power is $-3 \mathrm{dBm}$ for both formats. In Fig. 4(c), the measured BER as a function of transmission distance for the center of the WDM channels using the optimal launch power is shown for both 256- $\mathrm{D}_{4}$ and PM-16QAM. Plotted is both the uncoded case and the BER after iterative decoding of the TPC for both formats. At BER $=2 \times 10^{-2}$, the achievable transmission distance was $3100 \mathrm{~km}$ for uncoded PM-16QAM and $2500 \mathrm{~km}$ for $256-\mathrm{D}_{4}$. After decoding of the TPC, we observe no errors within the scope of our measurement systems for distances below $3360 \mathrm{~km}$ for PM-16QAM and $2400 \mathrm{~km}$ for 256- $\mathrm{D}_{4}$. For pre-FEC BER targets lower than $2.3 \times 10^{-3}, 256-\mathrm{D}_{4}$ has a longer reach, making it a format attractive for low cost/low complexity shorter reach systems.

\section{Conclusions}

We have experimentally compared 256- $\mathrm{D}_{4}$ to PM-16QAM and shown that in both back-to-back and in WDM longhaul transmission, PM-16QAM outperforms $256-\mathrm{D}_{4}$ when compared at a soft-decision FEC target of $\mathrm{BER}=2 \times 10^{-2}$. We also confirmed this by implementing the same TPC based FEC for both formats. For uncoded systems or lowcomplexity systems with a pre-FEC target below BER $=10^{-3}, 256-\mathrm{D}_{4}$ is a promising format, capable of increased transmission distances and showing a $0.9 \mathrm{~dB}$ better sensitivity than PM-16QAM measured at BER $=10^{-6}$.

\section{Acknowledgements}

This work was partly funded by the German Federal Ministry of Education and Research under support codes 16BP12310 and 16BP12402 (EUREKA-Project SASER) as well as the Swedish Research Council. Saleem Alreesh is funded by the German Research Foundation (DFG) under grant GR 3774/2-1.

\section{References}

[1] E. Agrell, et al., "Power-Efficient Modulation Formats in Coherent Transmission Systems," J. Lightwave Technol., vol. 27, pp. 5115-5126, 2009.

[2] J. K. Fischer, et al., "Bandwidth-Variable Transceivers based on Four-Dimensional Modulation Formats," J. Lightwave Technol., vol. 32 , pp. $2886-2895$, 2014.

[3] T. A. Eriksson, et al., "Comparison of 128-SP-QAM and PM-16QAM in Long-haul WDM Transmission," Opt. Exp., vol. 21, pp. 19269-19279, 2013.

[4] M. Karlsson, et al., "Spectrally Efficient Four-Dimensional Modulation,” OFC'12, Paper OTu2C.1., 2012.

[5] J. K. Fischer, et al., "Transmission of 512SP-QAM Nyquist-WDM Signals", ECOC'14, Paper Tu.3.3.2, 2014.

[6] G. Welti, et al.,"Digital Transmission with Coherent Four-Dimensional Modulation” IEEE Trans. Inf. Theory, vol. 20, pp. 497-502, 1974.

[7] J. Conway, et al., "A Fast Encoding Method for Lattice Codes and Quantizers", IEEE Trans. Inf. Theory, vol. 29, pp. 820-824, 1983.

[8] A. Alvarado, et al., "Achievable Rates for Four-Dimensional Coded Modulation with a Bit-Wise Receiver", OFC'14, Paper M2C.1., 2014.

[9] H. Buelow, et al., "Experimental Performance of 4D Optimized Constellation Alternatives for PM-8QAM and PM-16QAM," OFC'14, Paper M2A.6., 2014.

[10] R. Elschner et al., "Experimental Demonstration of a Format-Flexible Single-Carrier Coherent Receiver Using Data-aided Digital Signal Processing", Opt. Exp, vol. 20, p. 28786-28791, 2012.

[11] R. M. Pyndiah, "Near-Optimum Decoding of Product Codes: Block Turbo Codes", IEEE Trans. Commun., vol. 46, p. 1003-1010, 1998. 